\title{
DIFFERENTIAL EMISSION MEASURE ANALYSIS OF A POLAR CORONAL HOLE DURING THE SOLAR MINIMUM IN 2007
}

\author{
M. HAHN ${ }^{1}$, E. LANDI ${ }^{2}$, AND D. W. SAVIN ${ }^{1}$ \\ ${ }^{1}$ Columbia Astrophysics Laboratory, Columbia University, MC 5247, 550 West 120th Street, New York, NY 10027, USA \\ ${ }^{2}$ Department of Atmospheric, Oceanic and Space Sciences, University of Michigan, Ann Arbor, MI 48109, USA \\ Received 2010 December 20; accepted 2011 May 8; published 2011 July 13
}

\begin{abstract}
We have performed a differential emission measure (DEM) analysis for a polar coronal hole observed during solar minimum in 2007. Five observations are analyzed spanning the coronal hole from the central meridian to the boundary with the quiet-Sun corona. The observed heights ranged from 1.05 to $1.20 R_{\odot}$. The analysis shows that the plasma is not strictly isothermal anywhere, but rather has a high-temperature component that extends up to $\log T(\mathrm{~K})=6.2-6.3$. The size and importance of this component depend on location, and its evolving magnitude with height marks the boundary between the coronal hole and the quiet corona, where it becomes dominant. The DEM of the coronal hole plasma below $\log T(\mathrm{~K})=6.0$ decreases faster with height than that of the high-temperature component. We discuss the possible nature of the high-temperature component. Our results highlight the potential limitations of isothermal analyses. Such methods actually measure a DEM-weighted average temperature and as a result can infer artificial temperature gradients. Assuming the gas is isothermal along the line of sight can also yield incorrect electron densities. By revealing structures along the line of sight, a DEM analysis can also be used to more reliably interpret electron temperature and density measurements.
\end{abstract}

Key words: methods: data analysis - Sun: corona

\section{INTRODUCTION}

Coronal holes are open field regions of the solar atmosphere characterized by a lower electron temperature and density than closed field regions such as streamers and active regions. They are largely unstructured except for plumes which extend radially outward along the magnetic field lines and have a higher density than the interplume plasma (Wilhelm et al. 1998; Del Zanna et al. 2003). Large coronal holes are found at the polar regions of the Sun during the minimum phase of the solar cycle, but they can be located anywhere on the solar disk during the maximum phase. Coronal holes are the source regions of the fast solar wind (Krieger et al. 1973; Zirker 1977). Thus, an empirical understanding of the thermal structure of coronal hole plasmas is important for developing theoretical models of solar wind heating and acceleration (e.g., Kohl et al. 2006).

Many authors have studied the temperature and temperature distributions of coronal hole plasmas. Reviews of the main results obtained in the pre-SOHO era can be found in Wilhelm et al. (1998), while more recent measurements are reviewed in Landi (2008). These measurements have converged toward a picture of a nearly isothermal plasma slightly colder than $1 \mathrm{MK}$ whose temperature increases slowly with height. More recent studies carried out with the Hinode/Extreme-ultraviolet Imaging Spectrometer (EIS) by Hahn et al. (2010) agree with this scenario. In the present work, we show that their observations are not entirely isothermal, and that this changes some of their inferred plasma properties.

Most of the measurements in the literature have been performed employing a line intensity ratio technique, either using lines from the same ion or from consecutive ionization stages of an element. When lines from multiple ions (of the same or of different elements) were available, more sophisticated methodologies were adopted. Spectra observed outside the solar disk have been studied with an emission measure (EM) loci method. Both the line ratio and EM-loci methods assume the plasma is isothermal. This approximation breaks down for on-disk spectra, where the entire temperature range of the solar atmosphere is present along the line of sight. The thermal structure of these spectra has been inferred using a differential emission measure (DEM) method.

When the isothermal condition is not valid, line ratio and EM-loci methods fail and provide only an indication of the average temperature along the line of sight. While useful as a rough estimate, such average temperatures do not give information on the temperature distribution of the coronal hole plasma. For example, Landi (2008) suggested that the apparent temperature increase with height might be due to the presence of unresolved hotter streamer structures along the line of sight rather than due to a true temperature gradient in the coronal hole, but the EM-loci analysis used did not provide a definitive conclusion. Also, Landi \& Klimchuk (2010) have shown that in the presence of (unavoidable) uncertainties in the measured intensities, the EM-loci analysis is unable to distinguish between a truly isothermal plasma and a multithermal plasma with a narrow thermal distribution. A DEM diagnostic technique can potentially provide a more accurate determination of the real thermodynamic state of coronal hole plasmas and of any variation with height. To the best of our knowledge, until now such an analysis has only been applied to on-disk spectra.

The aim of the present work is to determine the thermal structure of coronal holes above the transition region by applying a DEM diagnostic technique to the same off-disk coronal hole spectra analyzed with an EM-loci technique by Hahn et al. (2010). The rest of this paper is organized as follows. The observation is described in Section 2. In Section 3, we review the DEM analysis method we use. The results and uncertainties are presented in Sections 4 and 5 with a discussion in Section 6. A summary is given in Section 7.

\section{OBSERVATIONS}

The observations discussed here are the same as those analyzed in Hahn et al. (2010), which describes the data reduction and line fitting in more detail. The data are from EIS 
(Korendyke et al. 2006; Culhane et al. 2007) on board Hinode (Kosugi et al. 2007). Five observations were made with the $2^{\prime \prime}$ slit pointed at different longitudinal positions within the north polar coronal hole on 2007 November 16. They were centered at $X=-7^{\prime \prime}, 108^{\prime \prime}, 223^{\prime \prime}, 324^{\prime \prime}$, and $423^{\prime \prime}$ relative to the center of the Sun. The above limb portions of the $-7^{\prime \prime}, 108^{\prime \prime}$, and $223^{\prime \prime}$ observations are completely within the coronal hole. The above limb portions of the $324^{\prime \prime}$ and $423^{\prime \prime}$ observations are near the boundary of the coronal hole and appear to include some quiet-Sun corona at low radii, below about $1.05 R_{\odot}$ and $1.15 R_{\odot}$, respectively. For each pointing the $2^{\prime \prime} \times 512^{\prime \prime}$ slit was rastered across seven positions in the horizontal direction giving a $14^{\prime \prime} \times 512^{\prime \prime}$ field of view. The DEM analysis was performed only for those pixels above the solar limb.

To improve statistics the data were binned into 10 vertical pixel by 7 horizontal pixel bins $\left(14^{\prime \prime} \times 10^{\prime \prime}\right)$. Gaussians were fitted to the binned data to extract the emission line intensities. The lines used in the analysis here are listed in Table 1. Only low first ionization potential (FIP) elements were used in order to avert the need to account for the FIP effect on elemental abundances (Feldman \& Laming 2000). Using only low FIP elements implies that if there is an elemental abundance enhancement due to the FIP effect the magnitude of the DEM curve would be affected but its shape would not be changed.

As in Hahn et al. (2010), we follow the recommendations of the EIS team to remove the contribution of disk radiation scattered inside the EIS instrument that contaminates the observed line intensities. This subtraction procedure will be discussed in more detail in Section 5.

\section{DIFFERENTIAL EMISSION MEASURE ANALYSIS}

The intensity of a spectral line emitted by a transition from level $j$ to level $i$ of charge state $q$ for element $\mathrm{X}$ is given by

$$
I_{j i}=\frac{1}{4 \pi} \int G\left(T, n_{\mathrm{e}}\right) n_{\mathrm{e}}^{2} d h .
$$

Here, $T$ is the electron temperature and $d h$ lies along the line of sight. The $G_{j i}\left(T, n_{\mathrm{e}}\right)$ contribution function is defined as

$$
G_{j i}\left(T, n_{\mathrm{e}}\right)=\frac{n_{j}\left(\mathrm{X}^{+q}\right)}{n\left(\mathrm{X}^{+q}\right)} \frac{n\left(\mathrm{X}^{+q}\right)}{n(\mathrm{X})} \frac{n(\mathrm{X})}{n(\mathrm{H})} \frac{n(\mathrm{H})}{n_{\mathrm{e}}} \frac{A_{j i}}{n_{\mathrm{e}}}
$$

where $n_{j}\left(\mathrm{X}^{+q}\right) / n\left(\mathrm{X}^{+q}\right)$ is the relative population of the upper level $j$ for charge state $\mathrm{X}^{+q}, n\left(\mathrm{X}^{+q}\right) / n(\mathrm{X})$ is the relative abundance for charge state $q$ of element $\mathrm{X}, n(\mathrm{X}) / n(\mathrm{H})$ is the abundance of $\mathrm{X}$ relative to hydrogen, $n(\mathrm{H}) / n_{\mathrm{e}}$ is the hydrogen density relative to that of free electrons, and $A_{j i}$ is the radiative transition rate. To calculate $G_{j i}\left(T, n_{\mathrm{e}}\right)$ we use the ionization balance of Bryans et al. (2009), the elemental abundances of Asplund et al. (2009), and atomic data from CHIANTI (Dere et al. 1997, 2009). Level populations were calculated taking into account excitation from inelastic collisions with free electrons and protons, as well as photoexcitation from background photospheric blackbody radiation and depopulation from spontaneous emission.

The DEM $\phi(T)$ describes the amount of material along the line of sight with temperature between $T$ and $T+d T$ so that

$$
n_{\mathrm{e}}^{2}=\phi(T) \frac{d T}{d h}
$$

\begin{tabular}{|c|c|c|c|c|}
\hline \multirow{2}{*}{$\frac{\text { Ion }}{\mathrm{Mg} \text { VI }}$} & \multirow{2}{*}{$\frac{\lambda(\AA)^{\mathrm{a}}}{268.991}$} & \multicolumn{3}{|c|}{ Transition $^{\mathrm{a}}$} \\
\hline & & $2 s^{2} 2 p^{32} D_{3 / 2}$ & - & $2 s 2 p^{42} P_{1 / 2}$ \\
\hline \multirow{2}{*}{ Mg VI } & 270.391 & $2 s^{2} 2 p^{32} D_{5 / 2}$ & - & $2 s 2 p^{42} P_{3 / 2}$ \\
\hline & 270.400 & $2 s^{2} 2 p^{32} D_{3 / 2}$ & - & $2 s 2 p^{42} P_{3 / 2}$ \\
\hline Mg VII & 276.154 & $2 s^{2} 2 p^{23} P_{0}$ & - & $2 s 2 p^{33} S_{1}$ \\
\hline Si VI & 246.003 & $2 s^{2} 2 p^{52} P_{3 / 2}$ & - & $2 s 2 p^{62} S_{1 / 2}$ \\
\hline Si VII & 272.648 & $2 s^{2} 2 p^{43} P_{2}$ & - & $2 s 2 p^{53} P_{1}$ \\
\hline Si VII & 275.361 & $2 s^{2} 2 p^{43} P_{2}$ & - & $2 s 2 p^{53} P_{2}$ \\
\hline Si VII & 275.676 & $2 s^{2} 2 p^{53} P_{1}$ & - & $2 s 2 p^{53} P_{1}$ \\
\hline Si IX & 258.082 & $2 s^{2} 2 p^{2}{ }^{1} D_{2}$ & - & $2 s 2 p^{31} D_{2}$ \\
\hline Six & 258.371 & $2 s^{2} 2 p^{2} P_{3 / 2}$ & - & $2 s 2 p^{22} P_{3 / 2}$ \\
\hline Si $\mathrm{x}$ & 261.044 & $2 s^{2} 2 p^{2} P_{3 / 2}$ & - & $2 s 2 p^{2} P_{1 / 2}$ \\
\hline Si $x$ & 272.006 & $2 s^{2} 2 p^{2} P_{1 / 2}$ & - & $2 s 2 p^{2} S_{1 / 2}$ \\
\hline $\operatorname{Six}$ & 277.278 & $2 s^{2} 2 p^{2} P_{3 / 2}$ & - & $2 s 2 p^{22} S_{1 / 2}$ \\
\hline Fe VIII & 185.213 & $3 p^{6} 3 d^{2} D_{5 / 2}$ & - & $3 p^{5} 3 d^{2}\left({ }^{3} F\right)^{2} F_{7 / 2}$ \\
\hline Fe VIII & 186.599 & $3 p^{6} 3 d^{2} D_{3 / 2}$ & - & $3 p^{5} 3 d^{2}\left({ }^{3} F\right)^{2} F_{5 / 2}$ \\
\hline Fe VIII & 194.661 & $3 p^{6} 3 d^{2} D_{5 / 2}$ & - & $3 p^{6} 4 p^{2} P_{3 / 2}$ \\
\hline Fe VIII & 197.362 & $3 p^{6} 3 d^{2} D_{5 / 2}$ & - & $3 p^{5} 3 d^{2}\left({ }^{1} S\right)^{2} P_{3 / 2}$ \\
\hline Fe IX & 188.497 & $3 s^{2} 3 p^{5} 3 d^{3} F_{4}$ & - & $3 s^{2} 3 p^{4}\left({ }^{3} P\right) 3 d^{2} G_{5}$ \\
\hline Fe IX & 189.941 & $3 s^{2} 3 p^{5} 3 d^{3} F_{3}$ & - & $3 s^{2} 3 p^{4}\left({ }^{3} P\right) 3 d^{2}{ }^{3} G_{4}$ \\
\hline Fe IX & 197.862 & $3 s^{2} 3 p^{5} 3 d^{1} P_{1}$ & - & $3 s^{2} 3 p^{5} 4 p^{1} S_{0}$ \\
\hline $\mathrm{Fex}$ & 174.531 & $3 s^{2} 3 p^{52} P_{1 / 2}$ & - & $3 s^{2} 3 p^{4}\left({ }^{3} P\right) 3 d^{2} D_{5 / 2}$ \\
\hline Fe $x$ & 182.307 & $3 s^{2} 3 p^{52} P_{1 / 2}$ & - & $3 s^{2} 3 p^{4}\left({ }^{3} P\right) 3 d^{2} P_{3 / 2}$ \\
\hline $\mathrm{Fe} x$ & 184.537 & $3 s^{2} 3 p^{52} P_{3 / 2}$ & - & $3 s^{2} 3 p^{4}\left({ }^{1} D\right) 3 d^{2} S_{1 / 2}$ \\
\hline $\mathrm{Fe} x$ & 190.037 & $3 s^{2} 3 p^{52} P_{1 / 2}$ & - & $3 s^{2} 3 p^{4}\left({ }^{1} D\right) 3 d^{2} S_{1 / 2}$ \\
\hline Fe $x$ & 193.715 & $3 s^{2} 3 p^{52} P_{3 / 2}$ & - & $3 s^{2} 3 p^{4}\left({ }^{1} S\right) 3 d^{2} D_{5 / 2}$ \\
\hline \multirow{2}{*}{$\mathrm{Fe} x$} & 257.259 & $3 s^{2} 3 p^{52} P_{3 / 2}$ & - & $3 s^{2} 3 p^{4}\left({ }^{3} P\right) 3 d^{4} D_{5 / 2}$ \\
\hline & 257.263 & $3 s^{2} 3 p^{52} P_{3 / 2}$ & - & $3 s^{2} 3 p^{4}\left({ }^{3} P\right) 3 d^{4} D_{7 / 2}$ \\
\hline Fe XI & 180.408 & $3 s^{2} 3 p^{43} P_{2}$ & - & $3 s^{2} 3 p^{3}\left({ }^{4} S\right) 3 d^{3} D_{3}$ \\
\hline $\mathrm{Fe} \mathrm{XI}$ & 182.169 & $3 s^{2} 3 p^{43} P_{1}$ & - & $3 s^{2} 3 p^{3}\left({ }^{4} S\right) 3 d^{3} D_{2}$ \\
\hline Fe XI & 188.232 & $3 s^{2} 3 p^{43} P_{2}$ & - & $3 s^{2} 3 p^{3}\left({ }^{2} D\right) 3 d^{3} P_{2}$ \\
\hline Fe XI & 188.299 & $3 s^{2} 3 p^{43} P_{2}$ & - & $3 s^{2} 3 p^{3}\left({ }^{2} D\right) 3 d^{1} P_{1}$ \\
\hline Fe XI & 189.719 & $3 s^{2} 3 p^{43} P_{0}$ & - & $3 s^{2} 3 p^{3}\left({ }^{2} D\right) 3 d^{3} P_{1}$ \\
\hline Fe XII & 192.394 & $3 s^{2} 3 p^{34} S_{3 / 2}$ & - & $3 s^{2} 3 p^{2}\left({ }^{3} P\right) 3 d^{4} P_{1 / 2}$ \\
\hline Fe XII & 193.509 & $3 s^{2} 3 p^{34} S_{3 / 2}$ & - & $3 s^{2} 3 p^{2}\left({ }^{3} P\right) 3 d^{4} P_{3 / 2}$ \\
\hline \multirow{2}{*}{ Fe XII } & 195.119 & $3 s^{2} 3 p^{34} S_{3 / 2}$ & - & $3 s^{2} 3 p^{2}\left({ }^{3} P\right) 3 d^{4} P_{5 / 2}$ \\
\hline & 195.179 & $3 s^{2} 3 p^{32} D_{3 / 2}$ & - & $3 s^{2} 3 p^{2}\left({ }^{1} D\right) 3 d^{2} D_{3 / 2}$ \\
\hline Fe XIII & 202.044 & $3 s^{2} 3 p^{23} P_{0}$ & - & $3 s^{2} 3 p 3 d^{3} P_{1}$ \\
\hline \multirow{2}{*}{ Fe XIII } & 203.797 & $3 s^{2} 3 p^{23} P_{2}$ & - & $3 s^{2} 3 p 3 d^{3} D_{2}$ \\
\hline & 203.828 & $3 s^{2} 3 p^{23} P_{2}$ & - & $3 s^{2} 3 p 3 d^{3} D_{3}$ \\
\hline Fe XIII & 251.956 & $3 s^{2} 3 p^{23} P_{2}$ & - & $3 s 3 p^{33} S_{1}$ \\
\hline $\mathrm{Fe}$ XIV & 264.790 & $3 s^{2} 3 p^{2} P_{3 / 2}$ & - & $3 s 3 p^{22} P_{3 / 2}$ \\
\hline Fe XIV & 270.522 & $3 s^{2} 3 p^{2} P_{3 / 2}$ & - & $3 s 3 p^{22} P_{1 / 2}$ \\
\hline $\mathrm{Fe} x \mathrm{~V}$ & 284.163 & $3 s^{2} S_{0}$ & - & $3 s 3 p^{1} P_{1}$ \\
\hline
\end{tabular}

Table 1

Line List

Note. ${ }^{\text {a }}$ Wavelengths and transitions taken from CHIANTI (Dere et al. 1997, 2009).

The emission line intensity can then be written in terms of $\phi(T)$ by substituting this expression into Equation (1) giving

$$
I_{j i}=\frac{1}{4 \pi} \int G_{j i}\left(T, n_{\mathrm{e}}\right) \phi(T) d T .
$$

Throughout this paper, $T$ is given in units of $\mathrm{K}$ and $\phi(T)$ in units of $\mathrm{cm}^{-5} \mathrm{~K}^{-1}$.

For most transitions the contribution function depends much more strongly on temperature than density so that $G_{j i}\left(T, n_{\mathrm{e}}\right) \approx$ $G_{j i}(T)$. Therefore, $n_{\mathrm{e}}$ can be fixed in the analysis. Following Hahn et al. (2010) we used the density derived from the Fe VIII $185.21 \AA / 186.60 \AA$ line intensity ratio for all observations except the $423^{\prime \prime}$ observation where we used the (Fe XIII 203.79 $\AA$ + Fe XIII 203.82 $\AA$ ) $/$ Fe XIII 202.04 $\AA$ ratio. Hahn et al. (2010) showed that the inferred density varied by 


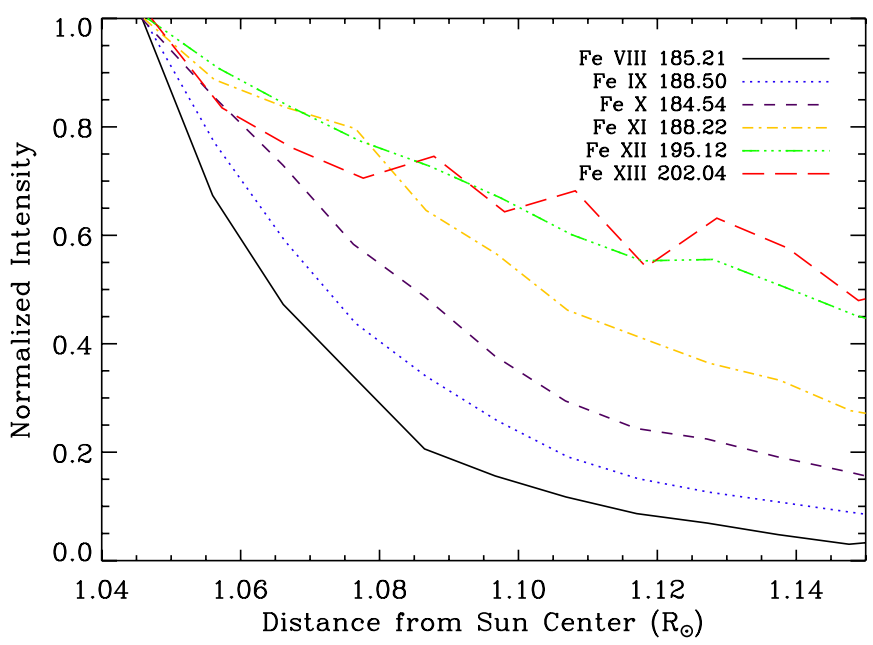

Figure 1. Normalized intensity profiles of lines emitted by consecutive stages of ionization of Fe. Intensities are normalized to the value at about $1.05 R_{\odot}$ and come from the $223^{\prime \prime}$ data set.

about a factor of two depending on the pair of lines used as a density diagnostic. This choice is discussed further in Section 5.

The observational data consist of intensities from a variety of lines with significantly different $G_{j i}(T)$ functions. In order to use these intensities to invert Equation (4) and solve for $\phi(T)$ we follow the iterative technique of Landi \& Landini (1997). For this technique, an initial trial DEM $\phi_{0}(T)$ is assumed. This DEM is related to the true $\phi(T)$ by a correction function $w(T)$ such that $\phi(T)=w(T) \phi_{0}(T)$. Landi \& Landini (1997) expand $w(T)$ about an effective temperature $\log T_{t}$. For each line, labeled by $j i$, the effective temperature is chosen to be

$$
\log T_{t}=\frac{\int G_{j i}(T) \phi_{0}(T) \log T d T}{\int G_{j i}(T) \phi_{0}(T) d T} .
$$

The measured intensity is then given by

$$
I_{j i}=\frac{1}{4 \pi} w\left(T_{t}\right) \int G_{j i}(T) \phi_{0}(T) d T
$$

where the choice of $\log T_{t}$ given by Equation (5) removes the first-order terms due to the expansion of $w(T)$ from Equation (6) and higher order effects will go to zero as $\phi(T)$ converges.

Using the known $G_{j i}(T)$, a trial $\phi(T)$, and the measured $I_{j i}$, the integrals can be calculated to find $T_{t}$ and $w\left(T_{t}\right)$ corresponding to each measured line. In order to smooth measurement uncertainties, the $w\left(T_{t}\right)$ are binned in temperature. For our analysis, bin sizes of $\Delta \log T=0.05-0.08$ were used. The function $\phi_{0}$ is then multiplied by the binned $w\left(T_{t}\right)$ factors to produce a corrected DEM $\phi_{1}$. Linear interpolation is used to extend $\phi_{1}$ at the bin centers to all $T$ and appropriate boundary conditions are applied to produce $\phi_{1}(T)$. The new DEM becomes the input for the next iteration. This procedure is repeated until $\phi(T)$ converges, i.e., $\phi_{n}(T) \approx \phi_{n-1}(T)$, which occurs when the binned $w\left(T_{t}\right)$ are all equal to one to within the estimated uncertainties of $\sim 30 \%$. These uncertainties are due to statistical errors in the measurement and fitting used to derive $I_{j i}$, possible unknown blends not accounted for in $G_{j i}(T)$, and atomic data errors in $G_{j i}(T)$. The iterative procedure will sometimes reach a point where $\phi(T)$ oscillates among several possible solutions. When this happens the analysis is repeated with a larger bin size until it converges to a single solution.

Since the solution for $\phi(T)$ is constrained only for a temperature range comparable to the range of formation temperatures

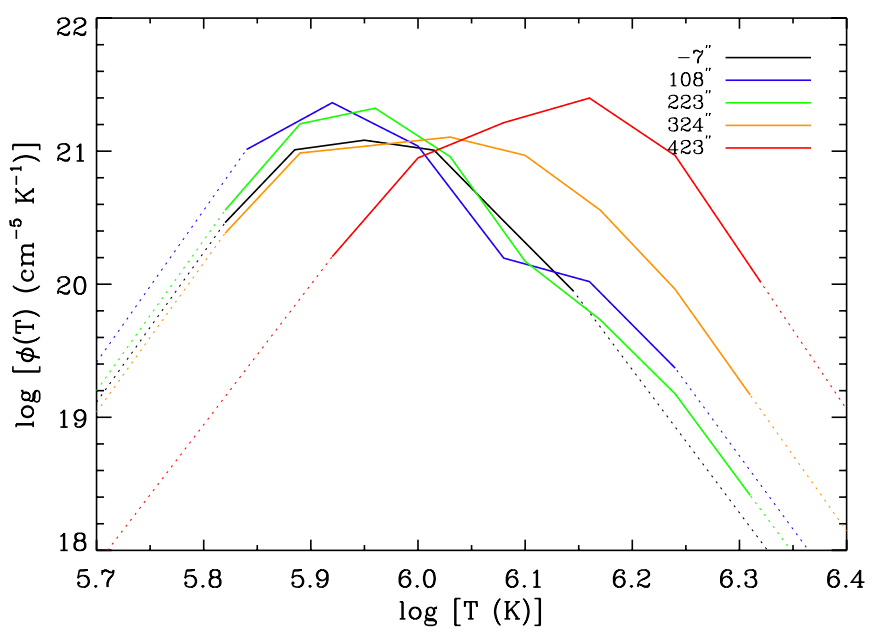

Figure 2. DEM $\phi(T)$ at $1.05 R_{\odot}$ is plotted for each pointing. The solid lines indicate the temperature range for which the DEM is constrained by the measured intensities and the dotted lines show the rest of the DEM as set by the boundary condition that $\phi(T)=0$ at $\log T(\mathrm{~K})=4$ and $\log T(\mathrm{~K})=8$.

for the observed lines it is necessary to apply boundary conditions to fix the DEM at higher and lower temperatures. For these off-limb observations $\phi(T)$ should go to zero at very high and very low temperatures. Thus, we arbitrarily require that $\phi(T)$ go to zero at $\log T=4.0$ and $\log T=8.0$. We discuss this choice in Section 5.

\section{RESULTS}

A simple inspection of the line intensities shows that the thermal structure of the observed gas is not constant with height even before reconstructing the DEM of the plasma. Figure 1 displays the normalized intensity profiles of lines from six consecutive stages of $\mathrm{Fe}$ shown as a function of distance from the limb. The intensities come from the 223" observation. If the plasma temperature were uniform with height the intensity profiles would overlap. Instead, Figure 1 shows that the line intensities decrease faster with height for decreasing Fe ionization stage. This behavior is consistent with a plasma temperature shifting toward higher values as the distance from the Sun increases. This can be due either to real heating of a locally isothermal coronal hole plasma, or to the increasing importance of hotter plasma, which in turn could be caused by intrinsically non-isothermal local coronal hole plasma, or to overarching streamer structures along the line of sight whose temperature is higher than that of the coronal hole.

In order to determine whether the plasma is really isothermal or not, we applied the diagnostic technique of Landi \& Klimchuk (2010), which allows one to determine whether a set of line intensities (with their uncertainties) can be reproduced by an isothermal plasma. We found that our data set fails the Landi \& Klimchuk (2010) test and is therefore not isothermal. Thus, multithermal plasma is present along the line of sight and the EM-loci method is inadequate for determining the structure of the plasma.

A more quantitative estimate of the overall change in temperature structure along the line of sight can be made by comparing $\phi(T)$ between the observations. First, we compare $\phi(T)$ curves obtained at the same height but at different EIS pointings. Figure 2 shows $\phi(T)$ at a height of $1.05 R_{\odot}$ for each pointing. The DEMs for the three pointings closest to the center of the polar coronal hole at $-7^{\prime \prime}, 108^{\prime \prime}$, and $223^{\prime \prime}$ are peaked at about 


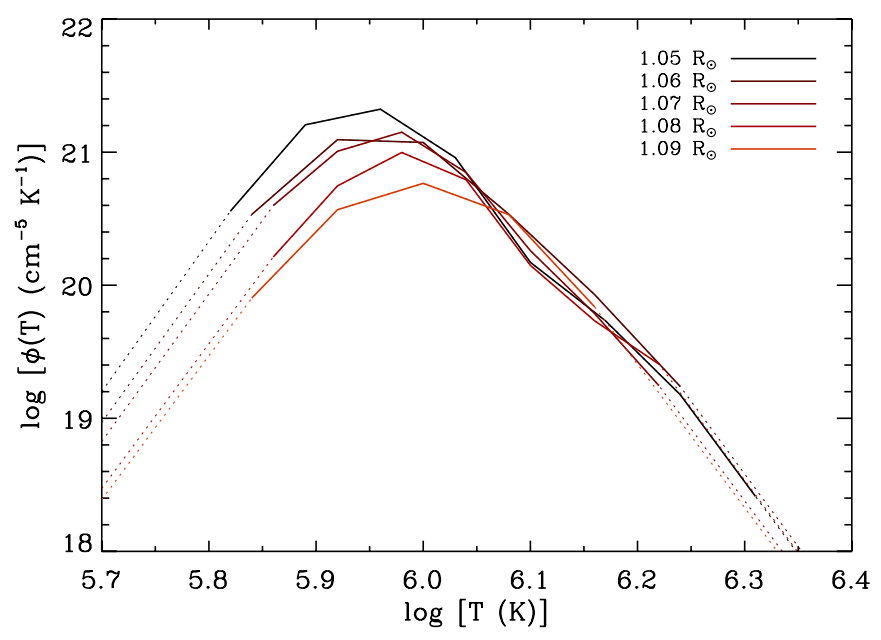

Figure 3. DEM $\phi(T)$ at different heights for the $223^{\prime \prime}$ observation. All else is the same as in Figure 2.

$\log T=5.95$, as expected for a coronal hole. They also show a high-temperature tail that extends to temperatures as high as $\log T=6.3$. This tail indicates that there is higher temperature material along the line of sight. The $324^{\prime \prime}$ observation looks at a region very close to the boundary of the coronal hole and the peak in the DEM is very broad. At this height the $423^{\prime \prime}$ observation looks into quiet-Sun corona and the DEM shows a peak at a higher temperature, $\log T=6.15$, typical of quiet-Sun off-disk temperatures. We interpret the broad peak in the $324^{\prime \prime}$ observation as a mixture of coronal hole and quiet-Sun plasma along the line of sight.

All together the peaks of all curves are consistent with other measurements obtained with other techniques from similar plasmas (e.g., Feldman et al. 1999; Fludra et al. 1999; Feldman \& Landi 2008; Landi 2008; Hahn et al. 2010). Additionally, the data are consistent with the temperatures hypothesized by Landi $\&$ Feldman (2008) to be characteristic of coronal holes and the quiet-Sun corona. More importantly, though, our measurements show that the plasma is not isothermal.

We next study the evolution of $\phi(T)$ with height. For each pointing, the magnitude of the DEM in different temperature ranges decreases with height at different rates. The altitude dependence for the $-7^{\prime \prime}, 108^{\prime \prime}$, and $223^{\prime \prime}$ observations is very similar and is illustrated in Figure 3 using the $223^{\prime \prime}$ observation as an example. There are two things to notice: the magnitude of the peak at $\log T=5.95$ decreases with height while the magnitude of the high-temperature component is almost constant with height. This is true even for the $-7^{\prime \prime}$ observation, which implies that there is higher temperature material along the line of sight in that observation even though it is not obvious in Figure 2. Figure 4 shows the 324" observation where $\phi(T)$ decreases more uniformly, but with the DEM at higher $T$ decreasing a little faster which causes a slight narrowing of the $\phi(T)$ curve with altitude. The faster decline of the DEM at higher temperatures is probably due to the boundary between the polar coronal hole and the quiet-Sun corona curving away from the vertical slit which puts the observation more solidly in the coronal hole at larger heights. In the $423^{\prime \prime}$ observation (Figure 5), the DEM decreases with height and maintains its shape until $1.11 R_{\odot}$, but beyond that limit the peak rapidly shifts to lower temperature and by $1.19 R_{\odot}$ the $\phi(T)$ resembles the curves of the $-7^{\prime \prime}, 108^{\prime \prime}$, and $223^{\prime \prime}$ pointings. We interpret this

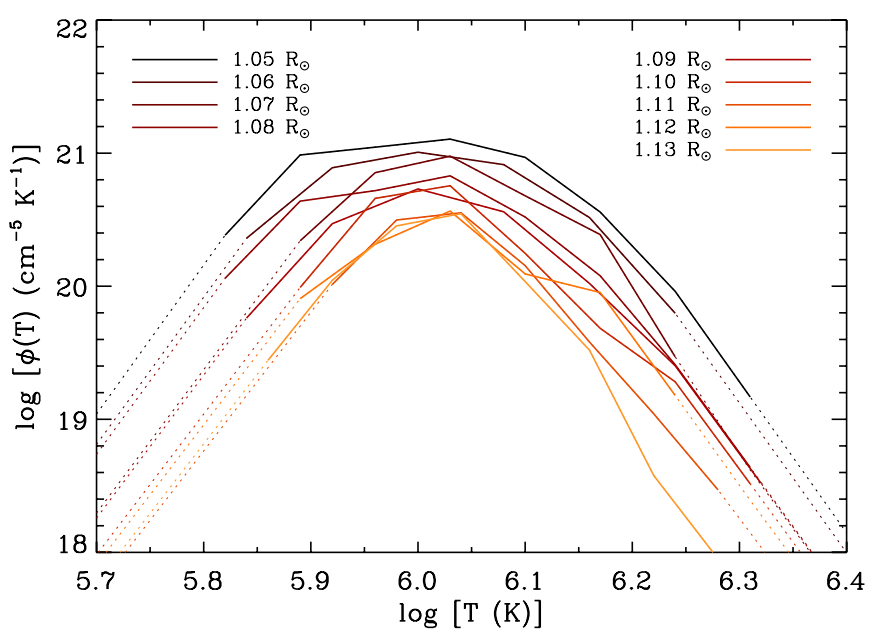

Figure 4. Same as Figure 3 but for the $324^{\prime \prime}$ observation.

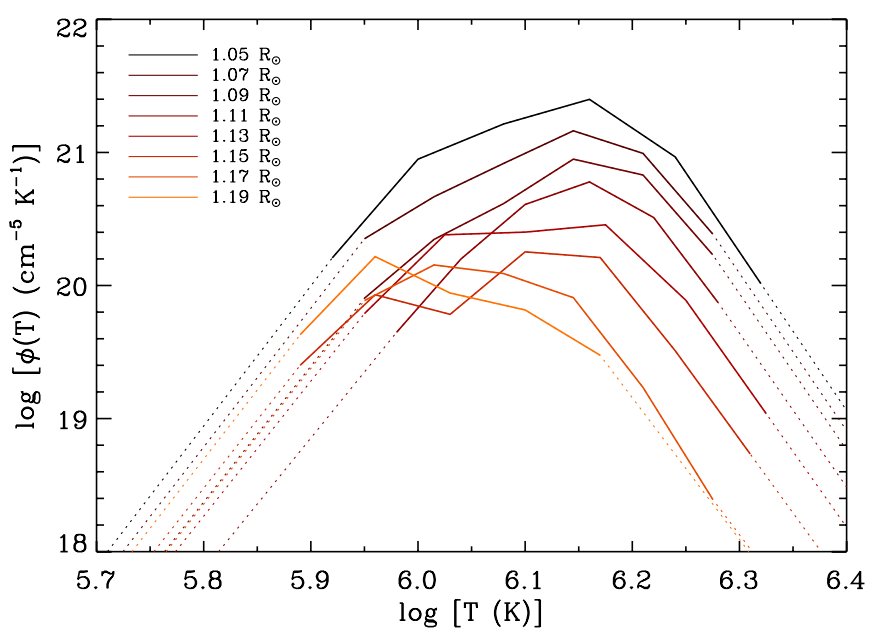

Figure 5. Same as Figure 3 but for the $423^{\prime \prime}$ observation.

as a transition from observing primarily quiet-Sun corona at low heights to the polar coronal hole at larger heights.

\section{UNCERTAINTIES}

DEM determinations are affected by a number of uncertainties. These can be grouped into three categories: intrinsic uncertainties in the diagnostic method, uncertainties in the atomic data and plasma parameters necessary to calculate level populations and line intensities, and uncertainties in the measured intensities due to counting statistics, assumed line profiles, calibration, and instrument scattered light. Unfortunately, it is impossible to develop an analytical way to propagate these uncertainties into the final results. But we can attempt to constrain the error in our results generated by these various uncertainties.

\subsection{DEM Reconstruction}

The significance of the DEM curves we obtained can be tested indirectly. Landi et al. (2011) carried out tests with a synthetic data set in order to ascertain the ability of a given DEM diagnostic technique to reproduce an arbitrary trial DEM. They tested the Monte Carlo Markov Chain (MCMC) technique developed by Kashyap \& Drake (1998). This technique was chosen because it is the only method that provides an estimate of the implicit uncertainty in the DEM reconstruction (e.g., Kashyap \& Drake 1998; Testa et al. 2011). The uncertainty is 


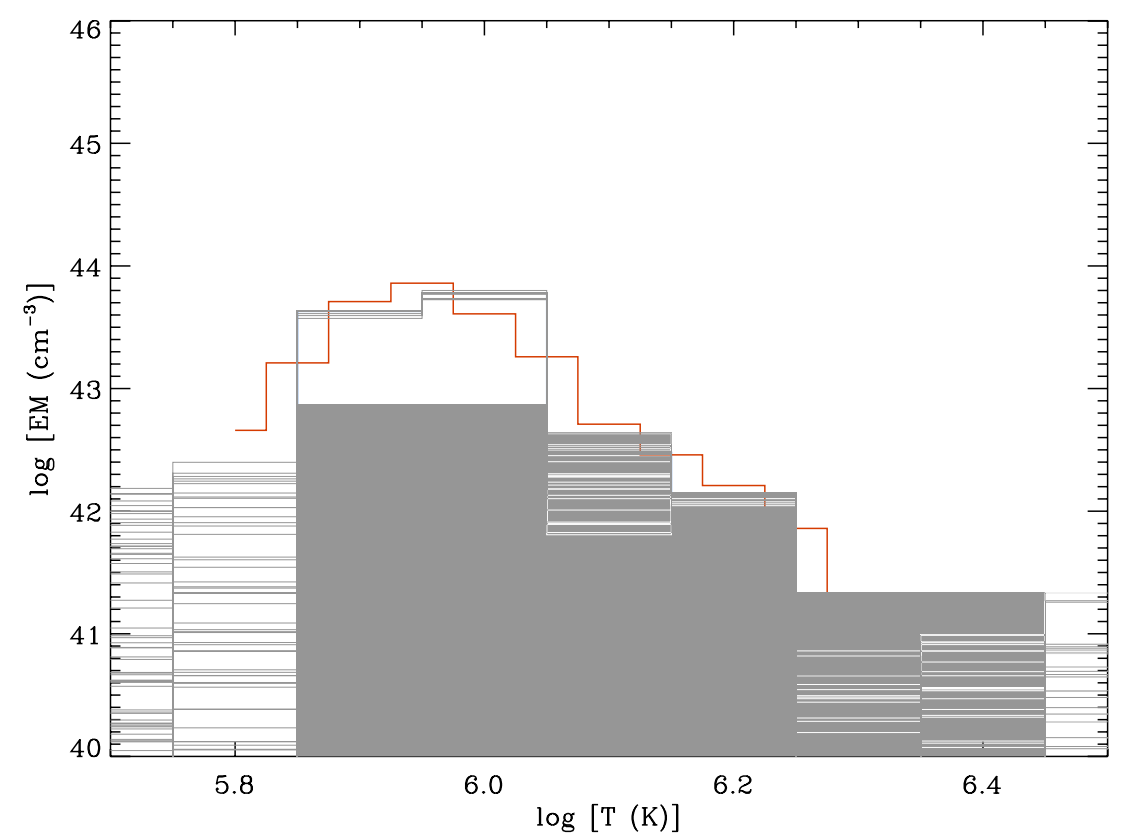

Figure 6. Comparison for the $223^{\prime \prime}$ observation at $1.05 R_{\odot}$ between the $\operatorname{EM}(T)$ curve obtained by integrating within each temperature bin the DEM determined with the Landi \& Landini (1997) technique (red curve) and the EM(T) curve determined with the MCMC diagnostic technique (Kashyap \& Drake 1998, blue curve). Gray curves indicate the range of MCMC solutions allowed by the data and provide an indication of the uncertainty in the results.

estimated from the range of EM values in each temperature bin obtained from a large number of runs. For the case of a multithermal plasma characterized by a continuous DEM function, the MCMC technique was able to reproduce well the original test DEM curve if a number of ions spanning a large temperature range were available. It is important to keep in mind that the MCMC approach is completely different from the Landi \& Landini (1997) method, so that we use the former here only to provide an estimate of the uncertainty and to check that both methods provide the same results. It is beyond the aim of the present work to compare these two methods and their characteristics.

In order to assess the reliability of our results and the uncertainties in the DEM, we applied the MCMC method to a subset of our data, specifically the $223^{\prime \prime}$ observation at $1.05 R_{\odot}$. We expect the MCMC method to provide reliable results because the set of lines and ions we used in this work spans from $\log T=5.63(\mathrm{Mg} \mathrm{vI})$ to $\log T=6.34$ (Fe Xv). This exceeds the range of temperatures typical of coronal holes and the quiet Sun. Figure 6 shows the comparison between our results using the Landi \& Landini (1997) method and the MCMC method. The MCMC technique divides the temperature range into a number of temperature bins and determines the value of the EM within each bin. Thus, in order to compare our DEM curve with the MCMC results, the former had to be interpolated into an arbitrary number of temperature bins, integrated in temperature, and adjusted by a constant factor involving the distance from the Sun in order to obtain the same units. Figure 6 shows that the two methods provide compatible results. The agreement is remarkable considering the intrinsic differences in the two diagnostic methods and in their temperature binning. In particular, both methods agree that the plasma is multithermal. The MCMC result seems also to show that the coronal hole plasma is made of two distinct components, one at typical coronal hole temperatures $(\log T=5.9-6.0)$ and the other at typical quiet-Sun temperatures $(\log T=6.2)$. The uncertainties for each temperature are rather tightly constrained. Thus, even if Figure 6 does not provide a quantitative estimate of the uncertainties in our results, it does give confidence that the multithermality of the plasma that we found is not an artifact of the Landi \& Landini (1997) method.

We also tested the choice of boundary conditions. We found that setting the high- and low-temperature limits of the DEM at $\log T=4.0$ and 8.0 does not affect the final results. These temperatures are far removed from the temperature range where the DEM is constrained by the data. In fact, moving these values to $\log T=5.0$ and 7.0 only changes the rate at which the extrapolated DEM decreases, but does not affect the shape and value of the DEM curve where line intensities directly contribute to its determination.

\subsection{DEM Resolving Power}

There are limits to the ability of a DEM method to reconstruct a truly isothermal DEM. In other words, a given DEM diagnostic technique cannot actually reconstruct a pure $\delta$-function isothermal DEM, but instead will produce a broadened DEM curve. Landi et al. (2011) recently investigated this for the MCMC method using spectral line intensities calculated with a DEM curve known a priori to reconstruct the original DEM. They found that a Gaussian DEM with a full width at half-maximum (FWHM) of $\Delta \log T=0.05$ or larger could be accurately reproduced. Also, the DEM reconstruction of an isothermal plasma provided identical results to a Gaussian DEM with FWHM $\Delta \log T=0.05$. Thus, the intrinsic resolution of that DEM diagnostic technique was estimated to be $\Delta \log T=0.05$. Given the similarity of the MCMC results to our results, we expect that the intrinsic resolution of the Landi \& Landini (1997) method is comparable.

We can constrain the resolution in our analysis by observing that the narrowest peaks in our DEM have an FWHM of $\Delta \log T=0.15$. This represents a combination of the intrinsic width, resolution broadening due to various uncertainties, plus any real broadening from a multithermal temperature 
distribution. Therefore, an FWHM of $\Delta \log T=0.15$ is an upper bound on the resolution of our results.

The high- and low-temperature components found in our results are separated by $\Delta \log T \approx 0.2$. These results lie outside the intrinsic DEM width. Therefore, we consider the shape of the DEM curves we measured to be indicative of the real multithermal structure of the plasma. This conclusion is also supported by the test discussed in Section 5.1, in which a different DEM diagnostic technique having different resolution characteristics reproduced the same major features in the DEM.

\subsection{Atomic Data}

The collision excitation rate coefficients used in the present work are taken from the CHIANTI database. Several benchmark studies have been carried out on these data as part of the CHIANTI project, and the ions that have been used in the present work were found to be in good agreement with observations (Young et al. 1998; Landi et al. 2002). Usually uncertainties in radiative decays and collisional excitation rates are believed to be of the order of $10 \%$ and $30 \%$, respectively. Landi et al. (2011) tested the effects of such uncertainties in DEM reconstructions and found that different levels of uncertainties did not affect significantly the results of the DEM reconstruction inside the range where spectral lines used for the analysis were formed, but could be large for temperatures outside that range.

\subsection{Density}

To test the effect of possible uncertainties in the density on the final DEM curve we repeated our analysis with other values for the density. These tests showed that changing the density by a factor of two produced a change in the solution $\phi(T)$ that was small compared to the difference in $\phi(T)$ between neighboring spatial bins. For example, in the temperature range $\log T=5.8-6.3$ for the $223^{\prime \prime}$ observation at $1.07 R_{\odot}$ the average absolute differences between $\log \phi(T)$ with $n_{\mathrm{e}}$ from the density analysis of Fe VIII and one-half or twice that value were 0.04 and 0.03 , respectively, but the absolute average difference between $1.07 R_{\odot}$ and $1.08 R_{\odot}$ was 0.17 . Thus, any potential factor of two variation in the density is not expected to have a significant effect on our DEM analysis.

\subsection{Intensity Calibration}

Intensity calibration is a key ingredient in any quantitative analysis of high-resolution spectra. There are two possible sources of uncertainty: absolute calibration and the relative calibration between different parts of the EIS wavelength range. Errors in the absolute calibration consist of a constant factor that applies to the entire spectrum. Its effect on DEM curves is to shift them by the same constant amount, while preserving their temperature dependence. Thus, absolute calibration errors are of no consequence to the present study.

Relative calibration errors could, in principle, affect DEM reconstructions. The EIS intensity calibration was determined before flight by first measuring the wavelength dependence of the response of each component of the EIS spectrometer in the laboratory, and then combining all the results together. This procedure has been thoroughly described by Lang et al. (2006) and Culhane et al. (2007). They found an overall uncertainty of $22 \%$, which includes both absolute and relative calibration. The EIS calibration has been monitored constantly since launch. The absolute calibration has been found to degrade over time, but there is no evidence for drifts in the relative calibration in any part of the EIS wavelength range. Thus, the uncertainty from the relative calibration is less than $22 \%$, which is at most about the same as the estimated atomic data uncertainties. In practice, the binning of the intensities from different ions by temperature combines lines from a different wavelengths and reduces any systematic effects from the relative calibration. We therefore expect errors from the relative intensity calibration to be small compared to other sources of uncertainty.

\subsection{Scattered Light}

As discussed in Hahn et al. (2010), the observed line intensities were corrected to remove contamination from instrumental scattered light. This was done by subtracting $2 \%$ of the average disk emission measured from the disk portion of the data from the intensities observed in the off-disk portion, as recommended by the EIS team (Ugarte-Urra 2010). This method can be a source of uncertainty if the $2 \%$ value we adopted is inaccurate or if the average disk emission from the small field of view of the EIS observation is not representative of the true disk emission.

We checked the validity of the $2 \%$ value for the scattered light by studying how the intensity ratio of the He II $256.3 \AA$ and the Si x 261.0 $\AA$ lines changed across the limb. The $256.3 \AA$ line is actually a blend of the He II line and a Si x line that is emitted by the same upper level as the $261.0 \AA$ line. Thus, the ratio of the two $\mathrm{Six}$ lines is determined only by the branching ratio and is constant. Inside the disk the He II line dominates the $256.3 \AA$ feature, but outside the disk the He II emission should be negligible and thus the $256.3 \AA / 261.0 \AA$ intensity ratio should approach the fixed ratio of the two Six lines (plus a small correction due to some blended Fe coronal lines). Any departure from this expectation must be due to He II intensity scattered from the disk. We found that once $2 \%$ of the disk emission is removed from the off-disk line intensities the $256.3 \AA$ / $261.0 \AA$ intensity ratio agreed to within the uncertainties with the expected ratio for the two Six lines at the heights where the two lines were visible. This validates the $2 \%$ value for the scattered light correction.

In order to assess the effect of misestimating the average disk intensity, we determined the contribution of the scattered light to the off-disk intensity. We found that scattered light, averaged over all lines, makes up less than $10 \%$ of the total intensity at heights lower than $1.10 R_{\odot}$. At larger heights the contribution rises to about $25 \%$ at $1.15 R_{\odot}$ and $40 \%$ at $1.20 R_{\odot}$. Thus, for heights below $1.10 R_{\odot}$, the uncertainty from the scattered light subtraction due to up to a factor of two uncertainty in the disk intensity is small compared to other sources of uncertainty.

\section{DISCUSSION}

\subsection{Thermal Structure of the Coronal Hole}

For the three pointings within the coronal hole at $-7^{\prime \prime}, 108^{\prime \prime}$, and $223^{\prime \prime}$, our results show that a high-temperature component is present at all heights and that this component decreases with height much more slowly than the peak at $\log T=5.95$. None of the observations is isothermal at any position. There are several different possible explanations for these results.

First, multithermality could be due to the presence of a locally isothermal plasma whose temperature increases with height. This is because the line of sight includes a range of heights from the lowest point above the photosphere, which we use to label the height positions, all the way to the instrument itself. Thus, if there is a temperature gradient, then even if the plasma 
is locally isothermal the line of sight is multithermal. If the gradient is such that the temperature increases with height, then the effect is to produce a high-temperature tail in the DEM.

We tested this scenario by estimating the contribution of each segment along the line of sight to the total intensity, which is proportional to the integral of $n_{\mathrm{e}}^{2}$. The scale height for coronal plasma at the temperature of the peak in our DEM of $\log T=5.95$ is $\approx 0.06 R_{\odot}$. Using this value for the scale height and integrating along the line of sight we found that $\approx 93 \%$ of the line intensity is emitted within $0.05 R_{\odot}$ of the nominal height, and $\approx 99 \%$ is emitted within $0.10 R_{\odot}$ of the nominal height. Thus, a large temperature increase of $\Delta \log T \sim 0.2$ over less than $0.1 R_{\odot}$ would be required to explain the high-temperature tail seen in our results under this scenario. Such a large gradient is incompatible with coronal hole temperature measurements (David et al. 1998; Wilhelm et al. 1998; Landi 2008; Hahn et al. 2010). Thus, this scenario cannot explain our results.

The second scenario is that the EIS slit observed both coronal hole and quiet-Sun plasma, each close to isothermal, the latter belonging to streamer structures rooted at lower latitudes and whose temperature is typically $\log T=6.1-6.2$. In this case the two plasmas are completely separated. The difference in evolution with height is caused by the smaller scale height of the cooler coronal hole plasma. However, one possible problem with this scenario is that if the height of the overarching structures containing quiet-Sun plasma is large, then $\phi(T)$ at temperatures larger than $\log T=6.1$ should also decrease consistent with the scale height. Such a decrease should be larger than what is actually observed. For example, the scale height for $\log T=5.95$ gas is $\approx 0.06 R_{\odot}$ and for $\log T=6.15$ is $\approx 0.1 R_{\odot}$. Thus, at $\log T=5.95$ the decrease of $\log \phi$ between $1.05 R_{\odot}$ and $1.09 R_{\odot}$ is expected to be about $0.6 \mathrm{~cm}^{-5} \mathrm{~K}^{-1}$, while for $\log T=6.15$ gas the decrease should be about $0.3 \mathrm{~cm}^{-5} \mathrm{~K}^{-1}$. The falloff of $\log \phi$ expected from the scale height is consistent with observations for the cooler gas, which drops by $0.5-0.7 \mathrm{~cm}^{-5} \mathrm{~K}^{-1}$ over this height range for these three pointings. However, at $\log T=6.15$ no decreasing trend was found.

A third scenario envisions the presence of distinct structures at different temperatures inside the coronal hole itself. For the $-7^{\prime \prime}, 108^{\prime \prime}$, and $223^{\prime \prime}$ observations, these may be coronal loops if the relative population changes with height so that hotter loops are more abundant at larger heights. Such a scenario is compatible with the multiple-component solar atmosphere proposed by Feldman \& Landi (2008), whereby the entire solar upper atmosphere is populated by nearly isothermal plasma structures whose temperature can have only a few values, and different regions (quiet Sun, coronal hole, and non-flaring active regions) are characterized by the amount of each structure present. In this scenario, coronal holes would be dominated by those plasma structures that have $\log T=5.95$, with a smaller number of $\log T=6.15-6.20$ structures. Such a scenario is also compatible with the results obtained at $X=324^{\prime \prime}$ and $423^{\prime \prime}$, assuming that the relative populations of hotter and colder loops change when the boundary between coronal hole and quiet Sun is crossed and those at $\log T=6.15-6.20$ prevail in quiet Sun. However, in this scenario a bi-modal $\phi(T)$ curve is expected, rather than the more continuous DEM curves obtained here. It is possible, though, that this bi-modal distribution is present but not resolved if the actual resolution of our results is close to the inferred upper bound of $\Delta \log T=0.15$.

Our results are not capable of clearly differentiating between the last two scenarios. They do show that the plasma is not strictly isothermal and that it has a high-temperature component which behaves differently with distance from the limb than the cooler component. However, they cannot provide a definitive answer as to the nature of the coronal plasma.

A significant limitation is that our DEM curves were obtained using lines from 14 ions with temperatures of formation in the range $\log T=5.6-6.3$. While typical of many spectroscopic observations, the number of ions in this data set is rather limited so that sharp variations of $\phi(T)$ over small temperature intervals, such as required by the bi-modal distribution of structures described above, would be lost. For comparison Landi \& Feldman (2008) used lines from 35 ions to determine that the plasma in an active region was made up of three distinct nearly isothermal components. For this reason, further studies are needed to unambiguously determine the nature of coronal hole plasmas. In particular, it will be important to use observations either with a larger signal to noise, or obtained with spectrometers working at different wavelength ranges, such as EIS and SOHO/SUMER, so that a larger number of ions formed at an even wider temperature range can be included in the analysis to improve the detail of the DEM reconstructions.

\subsection{On the Use of Isothermal Techniques}

Since the plasma is not isothermal, the temperatures inferred by Hahn et al. (2010) using an EM-loci technique are actually DEM-weighted averages. This effect is most clearly seen for the 324 " observation where Hahn et al. (2010) found $\log T \approx 6.05$. In contrast, the DEM analysis does not show any particular concentration of material at this temperature, but instead a single broad feature. The systematic effect of DEM-weighted averaging is less important for observations where one peak in $\phi(T)$ clearly dominates, as it does at low heights in the other four observations (see Figure 2).

The non-uniform change of $\phi(T)$ with height produces a false temperature gradient inferred in isothermal analyses. In the $-7^{\prime \prime}, 108^{\prime \prime}$, and $223^{\prime \prime}$ observations, as height increases $\phi(T)$ decreases more rapidly at low temperatures than at high temperatures. Isothermal analyses like line intensity ratios or EM-loci methods, which in fact measure a DEM-weighted average temperature, will therefore show an artificial temperature gradient with height (Doschek et al. 2001; Feldman \& Landi 2008; Hahn et al. 2010). To demonstrate this, we used the DEM curves for the $223^{\prime \prime}$ observation coupled with CHIANTI to generate emission line intensities by applying Equation (4). An isothermal temperature analysis was then applied to these synthetic intensities to derive the temperature from the O vi $173 \AA$ / $1032 \AA$ line intensity ratio and from an EM-loci method. The $\mathrm{O}$ VI ratio has been used by David et al. (1998) to measure the temperature of a coronal hole. Figure 7 compares these results to the DEM-weighted mean temperature, $\langle T\rangle=\int \phi(T) T d T / \int \phi(T) d T$, and to the EM-loci analysis results of Hahn et al. (2010). In each case there is an apparent temperature gradient due to the changing concentration of warm and cool plasma along the line of sight that matches the gradient in $\langle T\rangle$. Note that $\langle T\rangle$ does not have a physical significance: it only represents the temperature that would be inferred by applying an isothermal technique that in temperature uniformly samples the multithermal DEM. Also, our synthetic EM-loci result reproduces the observational results from Hahn et al. (2010) to within their uncertainties of about $\delta \log T \approx 0.05$.

The offsets in the magnitude of the EM-loci and $\mathrm{O}$ VI intensity ratio temperatures relative to $\langle T\rangle$ arise because those methods weight different parts of the multithermal DEM differently. The 


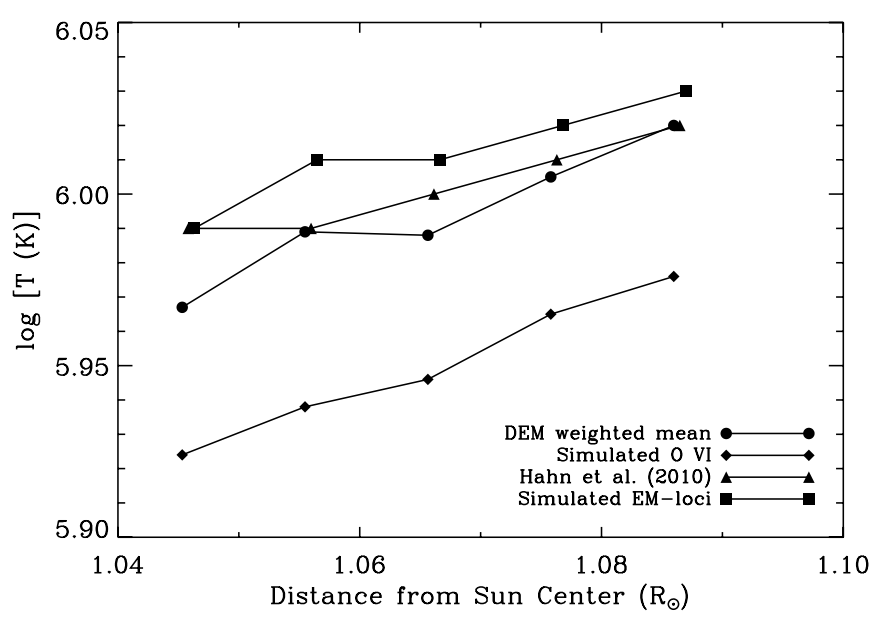

Figure 7. Results for various isothermal analyses applied to the $223^{\prime \prime}$ observation. The observationally inferred $\phi(T)$ curves were used to generate synthetic emission line intensities and calculate $T$ using an $\mathrm{O}$ vi line ratio diagnostic (diamonds) and the EM-loci technique (squares). These are compared to the DEM-weighted mean temperature (circles). Also shown are the EM-loci method results of Hahn et al. (2010, triangles). The isothermal analyses track the DEMweighted mean, but with offsets due to the different temperature regions sampled in $\phi(T)$ by the various methods (see the text for a more detailed explanation).

EM-loci technique is biased by the number of lines from each charge state used in the analysis. For example if more lines are formed at a high temperature, the EM-loci temperature result will be biased toward a high temperature also. The $\mathrm{O}$ VI temperature diagnostic is biased because for a multithermal plasma the $\mathrm{O}$ VI line emissivity samples a temperature range lower than most coronal lines. When calculating $\mathrm{O}$ vi line intensities with Equation (4), the lowest temperature range where the $\phi(T)$ curve is defined provides most of the simulated $\mathrm{O}$ VI line intensity because the ion fractional abundance of this ion peaks at $\log T \simeq 5.5$. Thus, the results of this method, applied to a multithermal plasma, tend to be biased toward this temperature.

The non-isothermal plasma distribution found here can affect not only temperature diagnostics but also the measurements of other properties. For example, Hahn et al. (2010) found that the inferred $n_{\mathrm{e}}$ differed systematically depending on the temperature of formation of the ions emitting the lines used as density diagnostics. They hypothesized that this discrepancy was caused by the emission coming from multiple structures along the line of sight having different temperatures and densities. We have attempted to verify this by using a DEM to model the intensities that would be observed from a multithermal plasma at different densities. In our crude model we took all gas cooler than some temperature $T_{0}$ to have density $n_{\mathrm{e}, 1}$ and warmer to have density $n_{\mathrm{e}, 2}$. Equation (4) can then be written as

$$
\begin{aligned}
I_{j i}= & \frac{1}{4 \pi}\left[\int_{0}^{T_{0}} G_{j i}\left(T, n_{\mathrm{e}, 1}\right) \phi(T) d T\right. \\
& \left.+\int_{T_{0}}^{\infty} G_{j i}\left(T, n_{\mathrm{e}, 2}\right) \phi(T) d T\right] .
\end{aligned}
$$

As an example, we take $\phi(T)$ from the $223^{\prime \prime}$ observation at $1.05 R_{\odot}$ and set $\log T_{0}=6.05$, which is approximately the boundary between coronal hole gas and quiet-Sun corona. We also use typical coronal hole and quiet-Sun densities of $n_{\mathrm{e}, 1}=0.8 \times 10^{8} \mathrm{~cm}^{-3}$ and $n_{\mathrm{e}, 2}=2 \times 10^{8} \mathrm{~cm}^{-3}$, respectively. Using CHIANTI, we then calculated the density from the line intensity ratios Fe VIII 185.21 $\AA / F e$ VIII $186.60 \AA$ and (Fe XIII
$203.79 \AA+$ Fe XIII 203.82 ̊)/ Fe XIII $202.04 \AA$. We infer that the density from the Fe VIII ratio is $1.0 \times 10^{8} \mathrm{~cm}^{-3}$ and from the Fe XIII ratio is $1.8 \times 10^{8} \mathrm{~cm}^{-3}$. These results show that the cooler lines, Fe VIII, probe the density of the cool coronal hole and the warmer lines, Fe XIII, probe the intervening quiet-Sun gas. This is consistent with the explanation of Hahn et al. (2010). However, neither set of lines provides a reliable density along the entire line of sight.

Density diagnostics through line intensity ratios assume that the plasma has the same density along the line of sight. The present test confirms the well known problem of line-of-sight contamination from multiple plasma structures with different densities, and is a warning toward using isothermal diagnostic results obtained in multiple-structure plasmas. Following Brown et al. (1991), a better determination of the electron density and temperature structure of an optically thin plasma can be obtained, in principle, by using a different definition of the DEM as $\psi\left(T, n_{\mathrm{e}}\right)$ incorporating the dependence on both quantities. An inversion technique can be applied to observed line intensities to determine $\psi\left(T, n_{\mathrm{e}}\right)$. However, the dependence of the emissivities of individual lines on $n_{\mathrm{e}}$ is much weaker than their temperature dependence, and it is restricted to only a limited density range. Thus, the results of such an inversion are very uncertain along the density dimension.

\section{SUMMARY}

A DEM analysis of a polar coronal hole observed during solar minimum has shown that the plasma along the line of sight was not isothermal in any of the regions we observed and a high-temperature component extending up to almost $2 \mathrm{MK}$ was present along the line of sight at all positions. In the coronal hole, this high-temperature component increased in importance relative to the DEM peak below $1 \mathrm{MK}$ as distance from the solar limb increased. We have discussed possible implications of the presence of such a high-temperature component. The shape of the DEM, and its variation with position and height above the limb, also allowed us to trace the boundary between the coronal hole and the quiet-Sun corona.

We have shown that temperature analysis methods relying on the assumption that the region is isothermal actually measure a DEM-weighted average temperature. For the observations considered here, an isothermal analysis overestimated the temperature of the polar coronal hole due to the contribution from the higher temperature gas. The isothermal analysis also found an artificial temperature gradient due to the changing ratio for the amount of high- to low-temperature gas at different heights in the observation. Electron density diagnostics can also be compromised by the isothermal assumption. Our work here demonstrates that isothermal analyses can have significant limitations even when applied to such relatively simple structures as polar coronal holes.

M.H. and D.W.S. were supported in part by the NASA Solar Heliospheric Physics program grant NNX09AB25G and the NSF Division of Atmospheric and Geospace Sciences SHINE program grant AGS-1060194. The work of E.L. is supported by the NASA grants NNX10AM17G and NNX11AC20G.

\section{REFERENCES}

Asplund, M., Grevesse, N., Sauval, A. J., \& Scott, P. 2009, ARA\&A, 47, 481 Brown, J. C., Dwivedi, B. N., Sweet, P. A., \& Almleaky, Y. M. 1991, A\&A, 249, 277 
Bryans, P., Landi, E., \& Savin, D. W. 2009, ApJ, 691, 1540

Culhane, J. L., et al. 2007, Sol. Phys., 243, 19

David, C., Gabriel, A. H., Bely-Dubau, F., Fludra, A., Lemaire, P., \& Wilhelm, K. 1998, A\&A, 336, L90

Del Zanna, G., Bromage, B. J. I., \& Mason, H. E. 2003, A\&A, 398, 743

Dere, K. P., Landi, E., Mason, H. E., Fossi, B. C. M., \& Young, P. R. 1997, A\&AS, 125, 149

Dere, K. P., Landi, E., Young, P. R., Zanna, G. D., Landini, M., \& Mason, H. E. 2009, A\&A, 498, 915

Doschek, G. A., Feldman, U., Laming, J. M., Schühle, U., \& Wilhelm, K. 2001, ApJ, 546, 559

Feldman, U., Doschek, G. A., Schühle, U., \& Wilhelm, K. 1999, ApJ, 518, 500

Feldman, U., \& Laming, J. M. 2000, Phys. Scr., 61, 222

Feldman, U., \& Landi, E. 2008, Phys. Plasmas, 15, 056501

Fludra, A., Zanna, G. D., Alexander, D., \& Bromage, B. J. I. 1999, J. Geophys. Res., 104, 9709

Hahn, M., Bryans, P., Landi, E., Miralles, M. P., \& Savin, D. W. 2010, ApJ, 725, 774

Kashyap, V., \& Drake, J. J. 1998, ApJ, 503, 450
Kohl, J. L., Noci, G., Cranmer, S. R., \& Raymond, J. C. 2006, A\&AR, 13, 31 Korendyke, C. M., et al. 2006, Appl. Opt., 45, 8674

Kosugi, T., et al. 2007, Sol. Phys., 243, 3

Krieger, A. S., Timothy, A. F., \& Roelof, E. C. 1973, Sol. Phys., 29, 505

Landi, E. 2008, ApJ, 685, 1270

Landi, E., \& Feldman, U. 2008, ApJ, 672, 674

Landi, E., Feldman, U., \& Dere, K. 2002, ApJ, 574, 495

Landi, E., \& Klimchuk, J. A. 2010, ApJ, 723, 320

Landi, E., \& Landini, M. 1997, A\&A, 327, 1230

Landi, E., Reale, F., \& Testa, P. 2011, ApJ, submitted

Lang, J., et al. 2006, Appl. Opt., 45, 8689

Testa, P., Reale, F., Landi, E., DeLuca, E., \& Kashyap, V. 2011, ApJ, 728, 30

Ugarte-Urra, I. 2010, EIS Software Note No. 12: https://hyperion.nascom.nasa gov/svn/eis/release/doc/eis_notes/12_STRAY_LIGHT/eis_swnote_12.pdf

Wilhelm, K., Marsch, E., Dwivedi, B. N., Hassler, D. M., Lemaire, P., Gabriel, A. H., \& Huber, M. C. E. 1998, ApJ, 500, 1023

Young, P., Landi, E., \& Thomas, R. 1998, A\&A, 329, 291

Zirker, J. B. (ed.) 1977, Coronal Holes and High-Speed Wind Streams (Boulder, CO: Colorado Assoc. Univ. Press) 